\title{
Stepping in the right direction but still some ways to go
}

\author{
Alfredo Addeo', Giuseppe Luigi Banna ${ }^{2}$, Alex Friedlaender ${ }^{1}$ \\ ${ }^{1}$ Oncology Department, Geneva University Hospital, Genève, Switzerland; ${ }^{2}$ Oncology Department, United Lincolnshire Hospital Trust, Lincoln, UK \\ Correspondence to: Alfredo Addeo. Oncology Department, Geneva University Hospital (CH), Switzerland. Email: alfredo.addeo@hcuge.ch. \\ Comment on: Shaw AT, Solomon BJ, Besse B, et al. ALK Resistance Mutations and Efficacy of Lorlatinib in Advanced Anaplastic Lymphoma Kinase- \\ Positive Non-Small-Cell Lung Cancer. J Clin Oncol 2019;37:1370-9.
}

Submitted Jul 03, 2019. Accepted for publication Aug 05, 2019.

doi: $10.21037 /$ tcr.2019.08.06

View this article at: http://dx.doi.org/10.21037/tcr.2019.08.06

Over the last few years we have witnessed substantial advances in systemic treatment of non-small cell lung cancer (NSCLC) patients harboring anaplastic lymphoma kinase gene rearrangements (ALK+ NSCLC). Multiple ALK inhibitors (ALKi) have been approved (alectinib, lorlatinib, brigatinib, ceritinib, crizotinib). Despite the survival benefit of all of these drugs, all patients with ALK+ NSCLC will inevitably progress at some point during their treatment. Given the increased number of therapeutic options, understanding the resistance mechanism appears to be increasingly important.

Recently, Shaw et al. (1) published the results of an ALK resistance mutation analysis, performed on either cellfree plasma DNA (cfDNA) or tissue DNA (tDNA) assays, in the NCT01970865 trial. In the phase II portion of the study, 228 NSCLC patients with ALK rearrangements were enrolled in one of several expansion cohorts defined by prior treatments, and were treated with lorlatinib (2). Primary endpoint of the study was to assess the overall and intracranial efficacy of lorlatinib. The results have led to the approval of lorlatinib in the United States and Japan, as well as a positive recommendation by the EMA, for previously treated, advanced ALK+ NSCLC. The secondary endpoint was to assess predictive markers of response to lorlatinib in tDNA and cfDNA.

Among all the patients, 45 (24\%) had one or more ALK mutations detected in cfDNA 41 (21\%) had no detectable mutations in cfDNA. Among the 191 archival and de novo tumour samples, 40 (24\%) harbored more than 1 ALK mutation. Within the de novo specimens, 76 (78\%) were adequate for NGS analysis of which 36 (47\%) were found to have one or more ALK mutations. No difference seen as far as types and incidences of ALK mutations was concerned in de novo tissue samples. What impacted on type and frequency of ALK mutations was the prior ALK TKI (ALKi) therapy. The patients who progressed on crizotinib (EXP2 to EXP3A), showed as the most common ALK mutations G1269A, F1174X, and L1196M. In the group treated with one or more second-generation TKIs (EXP3B to EXP5), the predominant ALK mutation was the G1202R deletion, detected in 53\% and 55\% of cfDNA and tDNA, respectively. The group of patients who progressed on crizotinib achieved a $73 \%$ objective response (ORR) to lorlatinib with a median progression free survival (PFS) of 11.1 months. Only 11 out of 44 patients (19\%) had detectable ALK mutations based on cfDNA genotyping. Irrespective of the genotyping analyses through which the mutation status was detected, the ORR was around $73-75 \%$. Furthermore in this group of crizotinib pre-treated patients, lorlatinib showed activity irrespective of the detection of an ALK mutation.

In the cohort of 139 patients (EXP3B to EXP5) who progressed after second-generation ALKi, the ORR was $40 \%$ (95\% CI, $32 \%$ to $49 \%$ ) with a median DOR of 7.1 months and PFS, 6.9 months. Only a $34(23 \%)$ patients had detectable ALK mutations, whereas 94 patients (71\%) did not through cfDNA analysis. The ORR to lorlatinib was different based on ALK mutation status: $62 \%$ ORR among mutation-positive patients and $32 \%$ among negative patients. This result was similar to what was seen in the tDNA analysis: ORRs was $69 \%$ and $27 \%$ in patients with and without an ALK mutation at progression, respectively. The median PFS was 7.3 and 5.5 months in patients with or without ALK mutations detected in cfDNA, respectively. 
However, both PFS and DOR were significantly longer in mutation-positive patients when tDNA analyses was performed: median PFS was 11.0 vs. 5.4 months in patients with or without ALK mutations respectively. Notably the median DOR was 24.4 vs. 4.3 in mutation-positive patients compared to mutation-negative ones.

This data showed that in patients who have previously received more than one second-generation ALKi, finding an ALK mutation in the tDNA or cfDNA could be predictive of longer and deeper benefit from third generation ALKi.

In conclusion lorlatinib has showed strong activity against all 5 of the most common ALK mutations with ORRs ranging from $42 \%$ to $89 \%$ irrespective of the genotyping assessment. The G1202R deletion was the most common mutation detected against which Lorlatinib was effective with 57\% ORR, 7.0 months median DOR and 8.2 months median PFS.

The efficacy of lorlatinib was also assesses in patients who harbored one or more ALK. This was exclusively done in the tDNA group due to the small number of samples in the cfDNA one. The ORR was higher among patients harboring 1 ALK mutation compared to those with more ALK mutations (75\% versus $56 \%$, respectively). Single ALK-mutation patients showed longer median DOR (24.4 months versus 6.1 months, respectively). Thus raising the hypothesis that lorlatinib might be more active in patients harboring a single ALK mutation post one or more second-generation ALKi.

The findings published by Shaw et al. (1) are therefore very intriguing: first of all, on the basis of cfDNA or tDNA genotyping, ALK mutation status post-crizotinib did not interfere or alternate the lorlatinib effectiveness : all the patients responded equally to lorlatinib irrespectively of the mutation status and the fact that was assessed by cfDNA or tDNA genotyping. The second aspect was that lorlatinib could remain active in patients who failed 1 or more second- progressed on crizotinib: harboring an ALK mutation seems to be predictor of response to lorlatinib. This suggests that finding ALK mutations may identify ALK-dependent tumors, but not necessarily to determine the right ALKi sequencing. On the other hand, patients without a detectable ALK mutation, are likely going to have an ALK-resistant tumor, raising the question of whether systemic chemotherapy should or might be considered in this contest. Pemetrexed-based chemotherapy has indeed proven to be effective (3) in ALK rearranged NSCLC patients and, although less effective than first or second line ALKi, given its RR ranging between $40 \%$ to $46 \%$, it may still play a role in ALK resistant tumors.

This study has the merit to have clarified the difference in the value, impact and relevance of detecting ALK mutations in tDNA and cfDNA post-crizotinib or post second generation ALKi. Detecting an ALK mutation post crizotinib will not have an impact on lorlatinib activity, but it will in case of progression after second generation ALKi. Thus in case of a ALK resistant tumor, pemetrexed based chemotherapy as alternative to a further ALKi ought to be considered. It would be interesting to explore whether at progression on chemotherapy an ALK resistant tumor could regain sensitivity to ALK. No robust data are available to guide us in this context.

Further trials are certainly warranted to identify and better understand the right ALKi treatment sequencing. We look forward to seeing and commenting the ALK Master Protocol trial (4) results which will likely elucidate many unanswered questions about the best ALKi treatment sequencing.

\section{Acknowledgments}

Funding: None.

\section{Footnote}

Provenance and Peer Review: This article is commissioned and reviewed by the Section Editor Wei Xu (Division of Respiratory Disease, Department of Geriatrics, the First Affiliated Hospital of Nanjing Medical University, Nanjing, China).

Conflicts of Interest: All authors have completed the ICMJE uniform disclosure form (available at http://dx.doi. org/10.21037/tcr.2019.08.06). Dr. Addeo, receiving honoraria and consulting fees from Roche, consulting fees and research funding from Boehringer Ingelheim, consulting fees from AstraZeneca, MSD Oncology, and Bristol-Myers Squibb, and travel and accommodation expenses from Roche, MSD Oncology, Boehringer Ingelheim, and Merck. Dr. Friedlaender, receiving honoraria from Roche, Pfitzer, Astella. Dr. Banna receiving honoraria honoraria for consulting (advisory board) from Merk Sharp \& Dohme, Boerhinger Ingelheim-Janssen Cilag and Roche.

Ethical Statement: The authors are accountable for all aspects of the work in ensuring that questions related 
to the accuracy or integrity of any part of the work are appropriately investigated and resolved.

Open Access Statement: This is an Open Access article distributed in accordance with the Creative Commons Attribution-NonCommercial-NoDerivs 4.0 International License (CC BY-NC-ND 4.0), which permits the noncommercial replication and distribution of the article with the strict proviso that no changes or edits are made and the original work is properly cited (including links to both the formal publication through the relevant DOI and the license). See: https://creativecommons.org/licenses/by-ncnd $/ 4.0 /$.

\section{References}

1. Shaw AT, Solomon BJ, Besse B et al. ALK Resistance

Cite this article as: Addeo A, Banna GL, Friedlaender A. Stepping in the right direction but still some ways to go. Transl Cancer Res 2019;8(Suppl 6):S603-S605. doi: 10.21037/ tcr.2019.08.06
Mutations and Efficacy of Lorlatinib in Advanced Anaplastic Lymphoma Kinase-Positive Non-Small-Cell Lung Cancer. J Clin Oncol 2019;37:1370-9.

2. Solomon BJ, Besse B, Bauer TM et al. Lorlatinib in patients with ALK-positive non-small-cell lung cancer: results from a global phase 2 study. Lancet Oncol 2018;19:1654-67.

3. Lee JO, Kim TM, Lee SH et al. Anaplastic lymphoma kinase translocation: a predictive biomarker of pemetrexed in patients with non-small cell lung cancer. J Thorac Oncol 2011;6:1474-80.

4. Biomarker/ALK Inhibitor Combinations in Treating Patients With Stage IV ALK Positive Non-squamous Non-small Cell Lung Cancer (The NCI-NRG ALK Protocol) NCT03737994. Available online: https:// clinicaltrials.gov/ct2/show/NCT03737994 